\title{
Einstein static universe on the brane supported by extended Chaplygin gas
}

\author{
Y. Heydarzade ${ }^{1}$, F. Darabi ${ }^{2}$ and K. Atazadeh ${ }^{3}$ \\ Department of Physics, Azarbaijan Shahid Madani University , Tabriz, 53714-161 Iran. \\ Research Institute for Astronomy and Astrophysics of Maragha (RIAAM), Maragha \\ 55134-441, Iran.
}

Received __; accepted _

\footnotetext{
1email: heydarzade@azaruniv.edu

${ }^{2}$ email: f.darabi@azaruniv.edu

${ }^{3}$ email: atazadeh@azaruniv.edu
} 


\begin{abstract}
We study the cosmological models in which an extended Chaplygin gas universe is merged with the braneworld scenario. In particular, we examine the realization of Einstein static universe on the brane embedded in a non-constant curvature bulk space and perform a detailed perturbation analysis. We extract the stability conditions and find their impacts on the geometric equation of state parameter and the spatial curvature of the universe.
\end{abstract}

Subject headings: Einstein static universe, Chaplygin gas, braneworld. 


\section{Introduction}

According to observations from different areas of cosmology, the universe has entered in a phase of accelerated expansion in the recent cosmological past (Riess et al 1998; Perlmutter et al 1999). Although the incorporation of a cosmological constant is the simplest explanation (peebles \& Ratra 2003), the possibility that the acceleration exhibit dynamical features led to two scenario.

In the first scenario, one can introduce the concept of dark energy, i.e. change the right hand side of the Einstein field equations. This can be realized by a quintessence field (Ratra \& Peebles 1988; Wetterich 1988; Liddle \& Scherrer 1999; Guo et al 2007; Dutta et al 2009), a phantom field (Caldwell 2002; Caldwell et al 2003; Nojiri \& Odintsov 2003;

Onemli \& Woodard 2004; Saridakis 2009), both fields as a quintum field(Guo et al 2005; Zhao 2006; Cai et al 2010), or more complex scenarios like K-essence (ArmendarizPicon et al 2001), Hordenski (Horndeski 1974), Galileons (Nicolis et al 2009; Deffavet et al 2009, 2011; Leon \& Saridakis 2013), holographic dark energy (Hooft 1993; Susskind 1995; Noiiri et al 2006; Bamba et al 2012), etc (for a review the reader is referred to (Copeland et all 2006)).

In the second scenario, one can introduce additional terms in the gravitational Lagrangian, that is modifying the gravitational theory, and consider the dark energy sector as an effective sector of gravitational origin. Specifically one can have the $f(R)$ gravity (DeFelice \& Tsujikawa 2010; Capozziello et al 2005; Amendola et al 2007; Capozziello et al 2011), the Gauss-Bonnet gravity (Wheeler 1986; Noiiri \& odintsov 2005; DeFelice \& Tsuiikawa 2009; Rastkar et al 2012), the Weyl gravity (Mannheim \& Kazanas 1989; Flanagan 2006), the Lovelock gravity (Lovelock 1971; Deruelle \& Farina-Busto 1990), the Hořava-Lifshitz gravity (Horava 2009; Calcagni 2009; Saridakis 2010), the nonlinear massive gravity terms (deRham et al 2011; Hinterbichler 2012; deRham 2014; Leon et al 2013), the $f(T)$ gravity (Bengochea \& Ferraro 2009; Linder 2010; 
Atazadeh \& Darabi 2012; Paliathanasis et al 2014) etc (for a reviews the reader is referred to (Capozziello \& De Laurentis 2011; Nojiri \& Odintsov 2006)). An interesting class of modified gravity also comes from the braneworld scenario, in which the universe is a brane embedded in a higher dimensional spacetime (Rubakov \& Shaposhnikov 1983; Randall \& Sundrum 1999; Darabi et al 2000; Brax \& van de Bruck 2003; Kovama 2008; Maeda et al 2007; Bazeia et al 2009; Setare \& Saridakis 2009; Lombriser et al 2009), however the gravitational interaction can act on the whole higher dimensional "bulk" space. Hence, the universe evolution is determined by the combination of the matter behavior on the brane, plus the effects if the higher dimensional gravity.

In the majority of cosmological models of the first scenario, the dark energy and dark matter belong to different sectors. However, one can propose scenarios where both these sectors are unified in a unique definition. This is indeed achieved by assuming that there is a unique cosmic fluid with an equation of state parameter of a Chaplygin gas type (Kamenshchik et al 2001; Bilic et al 2002; Gorini et al 2003) or its extensions (Bento et al] 2002; HeydariFard \& Sepangi| 2007; HevdariFard \$ Sepangi|2008; Ali et al 2012; Pourhassan 2013; Kahya \& Pourhassan 2014; Lu et al 2014; Pourhassan \& Kahya 2014), where at early times of universe evolution behaves as a pressureless fluid (matter-dark matter era), and at late times behaves like the cosmic fluid which mimics the cosmological constant (dark energy era).

In this work, we aim to study the cosmological models in which an extended Chaplygin gas universe, of the first scenario, is merged with the braneworld universe, of the second scenario. Moreover, motivated by the cosmological emergent universe scenario (Ellis \& Maartens 2004; Ellis et al 2004; Mukherjee et al 2006), where the big bang singularity is removed and the Universe is originated from an Einstein static state, we study the Einstein static universe and its stability in such a model. Similar 
attempts have already been done in the context of modified theories of gravity such as $f(R)$ (Barrow et al 1983; Bohmer et al 2007; Goswami et al 2008; Goheer et al 2009; Seahra \& Bohmer 2009), f(T) (Wu \& Yu 2011; Li \& Lee 2013), Einstein-Cartan theory (Bohmer 2004; Atazadeh 2014), massive gravity (Parisi et al 2012, 2014; Zhang et al 2013), loop quantum cosmology (Mulryne et al 2005; Parisi et al 2007; Canonico \& Parisi 2010), non-minimal kinetic coupled gravity (Atazadeh \& Darabi 2015), Horava-Lifshitz gravity

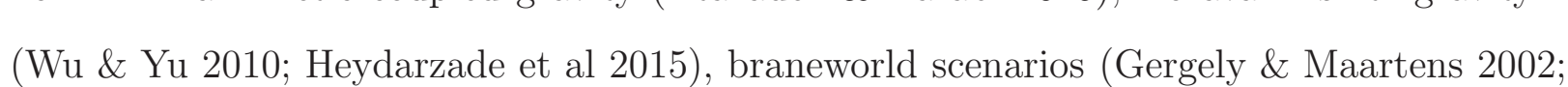
Zhang et al 2010, 2012; Clarkson \& Seahra 2005; Atazadeh et al 2014), induced matter theory (Hevdarzade \& Darabi 2015) Lyra geometry (Darabi et al 2014) and doubly general relativity(Khodadi et al 2015). We follow the approach of (Heydarzade et al 2015) and (Atazadeh et al 2014; Heydarzade \& Darabi 2015; Darabi et al 2014; Khodadi et al 2015) and extract the stability regions in terms of the geometric linear equation of state parameter. The plan of this work is as follows: In section 2, we present the geometrical setup of the model. In section 3, we perform a detailed analysis of the cosmological perturbations and focus on the Einstein static universe and extract the conditions for its stability. In section 4, we study some specific solutions. Finally, in section 5, we discuss on our results.

\section{General Geometrical Setup of the Model}

The effective Einstein-Hilbert action functional for the $4 D$ spacetime $\left(\mathcal{M}_{4}, g\right)$ embedded in a $n$-dimensional ambient space $\left(\mathcal{M}_{n}, \mathcal{G}\right)$ can be derived from the action

$$
I_{E H}=\frac{1}{2 \kappa_{n}^{2}} \int d^{n} x \sqrt{-\mathcal{G}} \mathcal{R}+\int_{\Sigma} d^{4} x \sqrt{-g} \mathcal{L}_{m}
$$

where $\kappa_{n}^{2}$ is the bulk space energy scale and $\mathcal{L}_{m}$ is the lagrangian of confined matter fields to the brane. The confinement hypothesis represents that the matter fields are trapped on the four dimensional brane. Variation of this action with respect to the ambient metric 
$\mathcal{G}_{A B}(A, B=0, \ldots, n-1)$ leads to the following Einstein field equations for the ambient space

$$
G_{A B}=8 \pi G_{n} S_{A B}
$$

where $G_{n}$ is the ambient gravitational constant and $S_{A B}$ is the matter energy-momentum tensor. Using the confinement hypothesis, we have

$$
8 \pi G_{n} S_{\mu \nu}=8 \pi G T_{\mu \nu}, \quad S_{\mu a}=0, \quad S_{a b}=0,
$$

where $a, b=4, \ldots, n-1$ labels the number of extra dimensions and $T_{\mu \nu}$ is the confined matter source on the brane.

For obtaining the effective Einstein field equation induced on the brane, we consider the following geometrical setup. Consider the $4 D$ background Lorentzian submanifold $\mathcal{M}_{4}$ isometrically embedded in a $n$ dimensional ambient space $\mathcal{M}_{n}$ by a differential map $\mathcal{Y}^{A}: \mathcal{M}_{4} \longrightarrow \mathcal{M}_{n}$ such that

$$
\mathcal{G}_{A B} \mathcal{Y}_{, \mu}^{A} \mathcal{Y}_{, \nu}^{B}=\bar{g}_{\mu \nu}, \quad \mathcal{G}_{A B} \mathcal{Y}_{, \mu}^{A} \overline{\mathcal{N}}_{a}^{B}=0, \quad \mathcal{G}_{A B} \overline{\mathcal{N}}_{a}^{A} \overline{\mathcal{N}}_{b}^{B}=g_{a b}
$$

where $\mathcal{G}_{A B}\left(\bar{g}_{\mu \nu}\right)$ is the metric of the ambient (brane) space $\mathcal{M}_{n}\left(\mathcal{M}_{4}\right)$ in which $\left\{\mathcal{Y}^{A}\right\}\left(\left\{x^{\mu}\right\}\right)$ is the basis of the ambient (brane), $\overline{\mathcal{N}}_{a}^{A}$ are $(n-4)$ normal unit vectors orthogonal to the brane and $g_{a b}=\epsilon \delta_{a b}$ in which $\epsilon= \pm 1$ represent the two possible signature of each extra dimension. Perturbation of the background submanifold $\mathcal{M}_{4}$ in a sufficiently small neighborhood of the brane along an arbitrary transverse direction $\xi^{a}$ is given by the following relation

$$
\mathcal{Z}^{A}\left(x^{\mu}, \xi^{a}\right)=\mathcal{Y}^{A}+\left(\mathcal{L}_{\xi} \mathcal{Y}\right)^{A}
$$

where $\mathcal{L}_{\xi^{a}}$ is the Lie derivative along $\xi^{a}$ where $\xi^{a}$ with $a=5, \ldots, n$ are small parameters along $\mathcal{N}^{A}{ }_{a}$ parameterizing the non-compact extra dimensions. By choosing the extra dimensions $\xi^{a}$ to be orthogonal to the brane, the gauge independency is guarantied (Nash 
1956; Jalazadeh \& Sepangi 2005) and we will have perturbations of the embedding along the orthogonal extra directions $\mathcal{N}_{a}^{A}$ which leads the local coordinates of the perturbed brane as

$$
\begin{aligned}
& \mathcal{Z}_{, \mu}^{A}\left(x^{\nu}, \xi^{a}\right)=\mathcal{Y}_{, \mu}^{A}\left(x^{\nu}\right)+\xi^{a} \mathcal{N}_{a, \mu}^{A} \\
& \mathcal{Z}_{, a}^{A}\left(x^{\nu}, \xi^{a}\right)=\mathcal{N}_{a}^{A} .
\end{aligned}
$$

It is seen from equation (5) that since the vectors $\mathcal{N}^{A}$ depend only on the local coordinates $x^{\mu}, \mathcal{N}^{A}=\mathcal{N}^{A}\left(x^{\mu}\right)$, they do not propagate along the extra dimensions of the ambient space and we have

$$
\mathcal{N}_{a}^{A}=\overline{\mathcal{N}}_{a}^{A}+\xi^{b}\left[\overline{\mathcal{N}}_{a}^{A}, \overline{\mathcal{N}}_{b}^{A}\right]=\overline{\mathcal{N}}_{a}^{A}
$$

These considerations give the embedding equations of the perturbed geometry as

$$
\mathcal{G}_{A B} \mathcal{Z}^{A}{ }_{, \mu} \mathcal{Z}^{B}{ }_{, \nu}=g_{\mu \nu}, \quad \mathcal{G}_{A B} \mathcal{Z}^{A}{ }_{, \mu} \mathcal{N}^{B}{ }_{a}=g_{\mu a}, \quad \mathcal{G}_{A B} \mathcal{N}^{A}{ }_{a} \mathcal{N}^{B}{ }_{b}=g_{a b} .
$$

where by setting $\mathcal{N}^{A}{ }_{a}=\delta^{A}{ }_{a}$, the metric of the ambient space $\mathcal{G}_{A B}$ in the vicinity of submanifold $\mathcal{M}_{4}$ and in the Gaussian frame can be written in the following matrix form

$$
\mathcal{G}_{A B}=\left(\begin{array}{cc}
g_{\mu \nu}+A_{\mu c} A_{\nu}^{c} & A_{\mu a} \\
A_{\nu b} & g_{a b}
\end{array}\right),
$$

which leads to the following line element for the ambient space

$$
d S^{2}=\mathcal{G}_{A B} d \mathcal{Z}^{A} d \mathcal{Z}^{B}=g_{\mu \nu}\left(x^{\alpha}, \xi^{a}\right) d x^{\mu} d x^{\nu}+g_{a b} d \xi^{a} d \xi^{b}
$$

where

$$
g_{\mu \nu}=\bar{g}_{\mu \nu}-2 \xi^{a} \bar{K}_{\mu \nu a}+\xi^{a} \xi^{b} \bar{g}^{\alpha \beta} \bar{K}_{\mu \alpha a} \bar{K}_{\nu \beta b},
$$

is the metric of the perturbed brane, or the first fundamental form, and

$$
\bar{K}_{\mu \nu a}=-\mathcal{G}_{A B} \mathcal{Y}_{, \mu}^{A} \mathcal{N}_{a ; \nu}^{B}=-\frac{1}{2} \frac{\partial g_{\mu \nu}}{\partial \xi^{a}}
$$


is the extrinsic curvature of the original brane, or the second fundamental form. We use the notation $A_{\mu c}=\xi^{d} A_{\mu c d}$ where

$$
A_{\mu c d}=\mathcal{G}_{A B} \mathcal{N}_{d ; \mu}^{A} \mathcal{N}_{c}^{B}=\bar{A}_{\mu c d}
$$

is known as the twisting vector fields, or the normal fundamental form. Any fixed $\xi^{a}$ denotes a new perturbed brane in which we can define an extrinsic curvature for this perturbed brane similar to the original one in the following form

$$
\tilde{K}_{\mu \nu a}=-\mathcal{G}_{A B} \mathcal{Z}^{A}{ }_{, \mu} \mathcal{N}_{a ; \nu}^{B}=\bar{K}_{\mu \nu a}-\xi^{b}\left(\bar{K}_{\mu \gamma a} \bar{K}^{\gamma}{ }_{\nu b}+A_{\mu c a} A^{c}{ }_{b \nu}\right)
$$

Note that the definitions (91), (11) and (14) require

$$
\tilde{K}_{\mu \nu a}=-\frac{1}{2} \frac{\partial \mathcal{G}_{\mu \nu}}{\partial \xi^{a}} .
$$

In the presence of gauge fields $A_{\mu a}$, the embedded family of submanifolds are tilted with respect to the normal vector $\mathcal{N}^{A}$. According to our geometrical construction, the original brane is orthogonal to the normal vector $\mathcal{N}^{A}$. However, the equation (8) shows that this is not the case for the deformed geometry. Then, we change the embedding coordinates to the following form

$$
\mathcal{X}_{, \mu}^{A}=\mathcal{Z}_{, \mu}^{A}-g^{a b} \mathcal{N}_{a}^{A} A_{b \mu},
$$

where the coordinates $\mathcal{X}^{A}$ describe a new family of embedded submanifolds whose members are always orthogonal to $\mathcal{N}^{A}$. In this coordinates the embedding equations of the perturbed brane is similar to the original one, represented by the equation (44), so that the coordinates $\mathcal{Y}^{A}$ is replaced by $\mathcal{X}^{A}$. The embedding of the local coordinates $\mathcal{X}^{A}$ are suitable than $\mathcal{Z}^{A}$ for obtaining the induced Einstein field equations on the brane. The extrinsic curvature of a perturbed brane in these coordinates, becomes

$$
K_{\mu \nu a}=-\mathcal{G}_{A B} \mathcal{X}_{, \mu}^{A} \mathcal{N}_{a ; \nu}^{B}=\bar{K}_{\mu \nu a}-\xi^{b} \bar{K}_{\mu \gamma a} \bar{K}_{\nu b}^{\gamma}=-\frac{1}{2} \frac{\partial g_{\mu \nu}}{\partial \xi^{a}},
$$


which is the generalized York's relation representing the propagation of the extrinsic curvature due to the metric propagation in the direction of extra dimensions. The components of the Riemann tensor of the ambient space in the embedding vielbein $\left\{\mathcal{X}_{, \alpha}^{A}, \mathcal{N}_{a}^{A}\right\}$, yield the Gauss-Codazzi equations (Eisenhart 1966) as

$$
\begin{gathered}
R_{\alpha \beta \gamma \delta}=2 g^{a b} K_{\alpha[\gamma a} K_{\delta] \beta b}+\mathcal{R}_{A B C D} \mathcal{X}_{, \alpha}^{A} \mathcal{X}_{, \beta}^{B} \mathcal{X}_{, \gamma}^{C} \mathcal{X}_{, \delta}^{D}, \\
2 K_{\alpha[\gamma c ; \delta]}=2 g^{a b} A_{[\gamma a c} K_{\delta] \alpha b}+\mathcal{R}_{A B C D} \mathcal{X}_{, \alpha}^{A} \mathcal{N}_{c}^{B} \mathcal{X}_{, \gamma}^{C} \mathcal{X}_{, \delta}^{D},
\end{gathered}
$$

where $\mathcal{R}_{A B C D}$ and $R_{\alpha \beta \gamma \delta}$ are the Riemann tensors of the ambient space and the perturbed brane, respectively. The Ricci tensor is obtainable by contracting the Gauss equation (18) as

$$
R_{\mu \nu}=\left(K_{\mu \alpha c} K_{\nu}{ }^{\alpha c}-K_{c} K_{\mu \nu}^{c}\right)+\mathcal{R}_{A B} \mathcal{X}_{, \mu}^{A} \mathcal{X}_{, \nu}^{B}-g^{a b} \mathcal{R}_{A B C D} \mathcal{N}_{a}^{A} \mathcal{X}_{, \mu}^{B} \mathcal{X}_{, \nu}^{C} \mathcal{N}_{b}^{D}
$$

The next contraction will give the Ricci scalar as

$$
R=\mathcal{R}+\left(K_{a \mu \nu} K^{a \mu \nu}-K_{a} K^{a}\right)-2 g^{a b} \mathcal{R}_{A B} \mathcal{N}_{a}^{A} \mathcal{N}_{b}^{B}+g^{a d} g^{b c} \mathcal{R}_{A B C D} \mathcal{N}_{a}^{A} \mathcal{N}_{b}^{B} \mathcal{N}_{c}^{C} \mathcal{N}_{d}^{D},
$$

where $K_{a} \equiv g^{\mu \nu} K_{a \mu \nu}$. Then, by using equations (20) and (21), we can obtain the following relation between the Einstein tensors of the ambient space and brane

$$
G_{A B} \mathcal{X}_{, \mu}^{A} \mathcal{X}_{, \nu}^{B}=G_{\mu \nu}-Q_{\mu \nu}-g^{a b} \mathcal{R}_{A B} \mathcal{N}_{a}^{A} \mathcal{N}_{b}^{B} g_{\mu \nu}+g^{a b} \mathcal{R}_{A B C D} \mathcal{N}_{a}^{A} \mathcal{X}_{\mu}^{B} \mathcal{X}_{\nu}^{C} \mathcal{N}_{b}^{D}
$$

where $G_{A B}$ and $G_{\mu \nu}$ are the Einstein tensors of the ambient space and brane respectively, and the new quantity $Q_{\mu \nu}$ as

$$
Q_{\mu \nu}=g^{a b}\left(K_{a \mu}^{\gamma} K_{\gamma \nu b}-K_{a} K_{\mu \nu b}\right)-\frac{1}{2}\left(K_{a \mu \nu} K^{a \mu \nu}-K_{a} K^{a}\right) g_{\mu \nu}
$$

is an independent conserved geometrical quantity, i.e. $\nabla_{\mu} Q^{\mu \nu}=0$ (Maia et al 2005).

Using the decomposition of the Riemann tensor of the ambient space into the Weyl curvature tensor, the Ricci tensor and the scalar curvature as

$$
\mathcal{R}_{A B C D}=C_{A B C D}-\frac{2}{n-2}\left(\mathcal{G}_{B[D} \mathcal{R}_{C] A}-\mathcal{G}_{A[D} \mathcal{R}_{C] B}\right)-\frac{2}{(n-1)(n-2)} \mathcal{R}\left(\mathcal{G}_{A[D} \mathcal{R}_{C] B}\right)
$$


we obtain the four dimensional induced Einstein equation on the brane as

$$
\begin{aligned}
G_{\mu \nu}= & G_{A B} \mathcal{X}_{, \mu}^{A} \mathcal{X}_{, \nu}^{B}+Q_{\mu \nu}-\mathcal{E}_{\mu \nu}+\frac{n-3}{n-2} g^{a b} \mathcal{R}_{A B} \mathcal{N}_{a}^{A} \mathcal{N}_{b}^{B} g_{\mu \nu} \\
& -\frac{n-4}{n-2} \mathcal{R}_{A B} \mathcal{X}_{, \mu}^{A} \mathcal{X}_{, \nu}^{B}+\frac{n-4}{(n-1)(n-2)} \mathcal{R} g_{\mu \nu},
\end{aligned}
$$

where $\mathcal{E}_{\mu \nu}=g^{a b} \mathcal{C}_{A B C D} \mathcal{X}_{, \mu}^{A} \mathcal{N}_{a}^{B} \mathcal{N}_{b}^{C} \mathcal{X}_{, \nu}^{D}$ is the electric part of the Weyl tensor of the ambient space $\mathcal{C}_{A B C D}$. The electric part of the Weyl tensor is well known from the brane point of view. It represents a traceless matter, denoted by dark radiation or Weyl matter where for a constant curvature ambient space, we have $\mathcal{E}_{\mu \nu}=0$.

Then, the induced Einstein equation in a non-constant curvature and Ricci flat ambient space (i.e. $\mathcal{E}_{\mu \nu} \neq 0$ and $\mathcal{R}_{A B}=0$ ) will be

$$
G_{\mu \nu}=T_{\mu \nu}+Q_{\mu \nu}-\mathcal{E}_{\mu \nu}
$$

where $T_{\mu \nu}$ is the confined matter source on the brane.

In a cosmological setup, for the purpose of embedding of the $F R W$ brane in a five dimensional ambient space, we consider the metric of

$$
d s^{2}=-d t^{2}+a(t)^{2}\left(\frac{d r^{2}}{1-k r^{2}}+r^{2} d \Omega^{2}\right)
$$

where $a(t)$ is the cosmic scale factor and $k=+1,-1$ or 0 corresponds to the closed, open or flat universes. The confined matter source on the brane $T_{\mu \nu}$ can be considered as a perfect fluid given in co-moving coordinates by

$$
T_{\mu \nu}=(\rho+p) u_{\mu} u_{\nu}+p g_{\mu \nu}
$$

where $u_{\alpha}=\delta_{\alpha}^{0}, \rho$ and $p$ are energy density and isotropic pressure, respectively. For the confined extended Chaplygin gas on brane, $p(t)$ has the form of

$$
p=\sum_{i=1}^{n} A_{i} \rho^{i}-\frac{B}{\rho^{\alpha}},
$$


where $A_{i}$ and $B$ are constants (Kahya \& Pourhassan 2014; Pourhassan \& Kahya 2014; Lu et al 2014). This model is reduced to generalized Chaplygin gas model introduced in (Kamenshchik et al 2001) and elaborated in (Bento et al 2002) with $A_{i}=0$. Also, it is reduced to the original Chaplygin gas scenario with $A_{i}=0$ and $\alpha=1$.

In order to obtain the components of $Q_{\mu \nu}$, we need to evaluate the components of the extrinsic curvature $K_{\mu \nu}$. Using the Codazzi equation, we obtain

$$
\begin{aligned}
K_{00} & =-\frac{1}{\dot{a}} \frac{d}{d t}\left(\frac{b}{a}\right), \\
K_{i j} & =\frac{b}{a^{2}} g_{i j}, i, j=1,2,3 .
\end{aligned}
$$

where dot denotes the derivative with respect to cosmic time $t$ and $b=b(t)$ is an arbitrary function (Maia et al 2005; Maia \& Roque 1989). By defining the new parameters $h:=\frac{\dot{b}}{b}$ and $H:=\frac{\dot{a}}{a}$ the components of $Q_{\mu \nu}$ represented by equation (23), will be

$$
\begin{aligned}
Q_{00} & =\frac{3 b^{2}}{a^{4}}, \\
Q_{i j} & =-\frac{b^{2}}{a^{4}}\left(\frac{2 h}{H}-1\right) g_{i j} .
\end{aligned}
$$

Similar to the confined source $T_{\mu \nu}$, the geometric energy-momentum tensor $Q_{\mu \nu}$ can be identified as a perfect fluid(Maia et al 2005)

$$
Q_{\mu \nu}=\left(\rho_{g}+p_{g}\right) u_{\mu} u_{\nu}+p_{g} g_{\mu \nu}
$$

where the $\rho_{g}$ and $p_{g}$ denoting the "geometric energy density" and "geometric pressure", respectively (the index $g$ stands for "geometric"). Then, using the equations (31) and (32), we will have

$$
\begin{aligned}
& \rho_{g}=\frac{3 b^{2}}{a^{4}}, \\
& p_{g}=-\frac{b^{2}}{a^{4}}\left(\frac{2 h}{H}-1\right) .
\end{aligned}
$$


Also, we consider the geometric fluid to have a barotropic equation of state $p_{g}=\omega_{g} \rho_{g}$ where $\omega_{g}$ is the geometric equation of state parameter and generally can be a function of time. Using equations (33) and the equation of state of the geometric fluid, we obtain the following equation for $b(t)$ in terms of the scale factor $a(t)$ and the equation of state parameter $\omega_{g}$ as

$$
\frac{\dot{b}}{b}=\frac{1}{2}\left(1-3 \omega_{g}\right) \frac{\dot{a}}{a}
$$

which cannot easily be solved because $\omega_{g}$ is not known. However, in the case of studying the Einstein static universe, a simple and useful consideration can be $\omega_{g}=\omega_{0 g}=$ constant leading to a general solution for the equation (34) as

$$
b=b_{0}\left(\frac{a}{a_{0}}\right)^{\frac{1}{2}\left(1-3 \omega_{0 g}\right)}
$$

where $a_{0}=$ constant is the scale factor of Einstein static universe and $b_{0}$ is an integration constant representing the curvature warp of this universe. Substituting equation (35) into equations (31) gives the geometric fluid component in terms of $b_{0}, a_{0}$ and $a(t)$ as

$$
\begin{aligned}
& Q_{00}(t)=\frac{3 b_{0}^{2}}{a_{0}^{1-3 \omega_{g}}} a^{-3\left(1+\omega_{g}\right)}, \\
& Q_{i j}(t)=3 \omega_{g} \frac{b_{0}^{2}}{a_{0}^{1-3 \omega_{g}}} a^{-3\left(1+\omega_{g}\right)} g_{i j},
\end{aligned}
$$

and consequently using equations (33), we get

$$
\begin{aligned}
& \rho_{g}(t)=\frac{3 b_{0}^{2}}{a_{0}^{1-\omega_{g}}} a^{-3\left(1+\omega_{g}\right)}, \\
& p_{g}(t)=3 \omega_{g} \frac{b_{0}^{2}}{a_{0}^{1-3 \omega_{g}}} a^{-3\left(1+\omega_{g}\right)} .
\end{aligned}
$$

For the Einstein static universe, $a=a_{0}=$ constant, the geometric fluid components are as follows

$$
\begin{aligned}
& Q_{00}\left(a_{0}\right)=\frac{3 b_{0}^{2}}{a_{0}^{4}} \\
& Q_{i j}\left(a_{0}\right)=3 \omega_{g} \frac{b_{0}^{2}}{a_{0}^{4}} g_{i j}
\end{aligned}
$$


Consequently, using equations (38), the geometric energy density and isotropic pressure will be

$$
\begin{aligned}
& \rho_{0 g}=\rho_{g}\left(a_{0}\right)=\frac{3 b_{0}^{2}}{a_{0}^{4}} \\
& p_{0 g}=p_{g}\left(a_{0}\right)=\frac{3 \omega_{g} b_{0}^{2}}{a_{0}^{4}} .
\end{aligned}
$$

Using equations (37) and (32), the induced Einstein equation on the brane (26) give us the following equation for the confined energy density

$$
\rho(t)=3\left(\frac{\dot{a}}{a}\right)^{2}+\frac{3 k}{a^{2}}-\frac{3 b_{0}^{2}}{a_{0}^{1-3 \omega_{g}}} a^{-3\left(1+\omega_{g}\right)}+\frac{\mu}{a^{4}},
$$

where $\mu$ is an integration constant which mathematically can be positive or negative depending on the geometry of the bulk (Mukohyama 1999). The standard big-bang cosmology does not include the third and fourth terms in the right hand of equation (40). The third term comes from the extrinsic geometry of the embedded brane through the quantity $Q_{\mu \nu}$. The fourth term which scales just like as the radiation with a constant $\mu$, is known as the dark radiation arising from the electric part of the Weyl tensor of the ambient space $\mathcal{E}_{\mu \nu}$. Both positive and negative values for $\mu$ are possible mathematically. On the other hand, dark radiation has influence on both of the big-bang nucleosynthesis and the cosmic microwave background. Then, one can determine both the magnitude and sign of the dark radiation using the constraints coming from the observations related to the big-bang nucleosynthesis and the cosmic microwave background (Ichiki et al 2002; Langlois et al 2001). For the Einstein static universe, the equation (40) takes the following form

$$
\rho_{0}=\rho\left(a_{0}\right)=\frac{3 k}{a_{0}^{2}}-\frac{3 b_{0}^{2}}{a_{0}^{4}}+\frac{\mu}{a_{0}^{4}} .
$$

Similarly, the confined isotropic pressure component can be obtained from equations $(26),(32)$ and (37) as

$$
p(t)=-2 \frac{\ddot{a}}{a}-\left(\frac{\dot{a}}{a}\right)^{2}-\frac{k}{a^{2}}-\frac{3 b_{0}^{2} \omega_{g}}{a_{0}^{1-3 \omega_{g}}} a^{-3\left(1+\omega_{g}\right)}+\frac{\mu}{a^{4}},
$$


leading to

$$
\sum_{i=1}^{n} A_{i} \rho_{0}^{i}-\frac{B}{\rho_{0}{ }^{\alpha}}=-\frac{k}{a_{0}^{2}}-\frac{3 b_{0}^{2} \omega_{g}}{a_{0}^{4}}+\frac{\mu}{a_{0}^{4}},
$$

for the Einstein static universe.

\section{Perturbations and stability analysis of the Einstein static state}

In what follows, we consider linear homogeneous scalar perturbations around the Einstein static universe, given in equations (41) and (43), and explore its stability against

these perturbations. Thus, the perturbation in the cosmic scale factor $a(t)$ and the confined energy density $\rho(t)$ depend only on time can be represented by

$$
\begin{aligned}
& a(t) \rightarrow a_{0}(1+\delta a(t)), \\
& \rho(t) \rightarrow \rho_{0}(1+\delta \rho(t)) .
\end{aligned}
$$

Substituting these equations in the equation (40) with subtracting $\rho_{0}$ and linearizing the result give the following equation

$$
\rho_{0} \delta \rho(t)=\left(-\frac{6 k}{a_{0}^{2}}+\frac{9 b_{0}^{2}\left(1+\omega_{g}\right)-4 \mu}{a_{0}^{4}}\right) \delta a(t) .
$$

Similarly, one can consider a linear equation of state $p(t)=\omega \rho(t)$ for confined source.

Applying the same method in obtaining the equation (45), on the equations (42) and (43) we also get

$$
\left(\sum_{i=1}^{n} i A_{i} \rho_{0}{ }^{i-1}+\frac{\alpha B}{\rho_{0}{ }^{\alpha-1}}\right) \rho_{0} \delta \rho=-2 \delta \ddot{a}+\left(\frac{2 k}{a_{0}^{2}}+\frac{9 b_{0}^{2} \omega_{g}\left(1+\omega_{g}\right)-4 \mu}{a_{0}^{4}}\right) \delta a .
$$

Substituting equation (45) in (46) gives the equation

$$
\begin{array}{r}
\ddot{\delta a}+\frac{1}{2}\left[\left(-\frac{6 k}{a_{0}^{2}}+\frac{9 b_{0}^{2}\left(1+\omega_{g}\right)-4 \mu}{a_{0}^{4}}\right)\left(\sum_{i=1}^{n} i A_{i} \rho_{0}{ }^{i-1}+\frac{\alpha B}{\rho_{0}{ }^{\alpha-1}}\right)\right. \\
\left.-\left(\frac{2 k}{a_{0}^{2}}+\frac{9 b_{0}^{2} \omega_{g}\left(1+\omega_{g}\right)-4 \mu}{a_{0}^{4}}\right)\right] \delta a=0 .
\end{array}
$$


This equation has the solution

$$
\delta a=C_{1} e^{i \gamma t}+C_{2} e^{-i \gamma t}
$$

where $C_{1}$ and $C_{2}$ are integration constants and $A$ is given by

$\gamma^{2}=\frac{1}{2}\left[\left(-\frac{6 k}{a_{0}^{2}}+\frac{9 b_{0}^{2}\left(1+\omega_{g}\right)-4 \mu}{a_{0}^{4}}\right)\left(\sum_{i=1}^{n} i A_{i} \rho_{0}{ }^{i-1}+\frac{\alpha B}{\rho_{0}{ }^{\alpha-1}}\right)-\left(\frac{2 k}{a_{0}^{2}}+\frac{9 b_{0}^{2} \omega_{g}\left(1+\omega_{g}\right)-4 \mu}{a_{0}^{4}}\right)\right]$.

Then, for having oscillating perturbation modes representing the existence of a stable Einstein static universe, the following condition should be satisfied

$$
\left(-\frac{6 k}{a_{0}^{2}}+\frac{9 b_{0}^{2}\left(1+\omega_{g}\right)-4 \mu}{a_{0}^{4}}\right)\left(\sum_{i=1}^{n} i A_{i} \rho_{0}{ }^{i-1}+\frac{\alpha B}{\rho_{0}{ }^{\alpha-1}}\right)-\left(\frac{2 k}{a_{0}^{2}}+\frac{9 b_{0}^{2} \omega_{g}\left(1+\omega_{g}\right)-4 \mu}{a_{0}^{4}}\right)>0
$$

which can be rewritten as the following form for the geometric equation of state parameter

$$
\omega_{g}^{2}+\mathcal{L}_{1} \omega_{g}+\mathcal{L}_{2}<0
$$

where

$$
\begin{aligned}
& \mathcal{L}_{1}=1-\sum_{i=1}^{n} i A_{i} \rho_{0}{ }^{i-1}-\frac{\alpha B}{\rho_{0}{ }^{\alpha-1}} \\
& \mathcal{L}_{2}=\frac{2 k a_{0}^{2}-4 \mu}{9 b_{0}^{2}}-\left(\sum_{i=1}^{n} i A_{i} \rho_{0}{ }^{i-1}+\frac{\alpha B}{\rho_{0}{ }^{\alpha-1}}\right)\left(1-\frac{6 k a_{0}^{2}+4 \mu}{9 b_{0}^{2}}\right)
\end{aligned}
$$

The inequality (51) leads to the following acceptable range

$$
\omega_{g}^{(1)}<\omega_{g}<\omega_{g}^{(2)}
$$

where

$$
\begin{aligned}
& \omega_{g}^{(1)}=-\frac{1}{2} \mathcal{L}_{1}-\frac{1}{2} \sqrt{\mathcal{L}_{1}^{2}-4 \mathcal{L}_{2}} \\
& \omega_{g}^{(2)}=-\frac{1}{2} \mathcal{L}_{1}+\frac{1}{2} \sqrt{\mathcal{L}_{1}^{2}-4 \mathcal{L}_{2}} .
\end{aligned}
$$


It is seen that the following condition also should be satisfied

$$
\mathcal{L}_{1}^{2}-4 \mathcal{L}_{2} \geq 0
$$

which results in the class of solutions to be discussed in the following section.

\section{Some specific Solutions}

\subsection{The case of $B=0$ with $A_{i \geq 2}=0$}

By defining $A_{1}=\omega$, this case reduces to the barotropic equation of state with

$$
\begin{aligned}
& \omega_{g}^{(1)}=-\frac{1}{2}+\frac{1}{2} \omega-\frac{1}{2} \sqrt{(1+\omega)^{2}-\frac{8 k(1+3 \omega) a_{0}^{2}-16 \omega \mu}{9 b_{0}^{2}}}, \\
& \omega_{g}^{(2)}=-\frac{1}{2}+\frac{1}{2} \omega+\frac{1}{2} \sqrt{(1+\omega)^{2}-\frac{8 k(1+3 \omega) a_{0}^{2}-16 \omega \mu}{9 b_{0}^{2}}},
\end{aligned}
$$

where we can investigate this solution with more details by dividing in the following classes. In this case, we recover the results in (Atazadeh et al 2014) by considering a constant curvature bulk, i.e setting $\mu=0$.

\subsubsection{Vacuum dominated state $\omega=-1$.}

The corresponding acceptable range for $\omega_{g}$ is

$$
-1-\frac{2}{3} \sqrt{\frac{k a_{0}^{2}-\mu}{b_{0}^{2}}}<\omega_{g}<-1+\frac{2}{3} \sqrt{\frac{k a_{0}^{2}-\mu}{b_{0}^{2}}},
$$

where we also should have

$$
k a_{0}^{2}-\mu \geq 0
$$

Regarding the relation (58), it is seen that for this state to be stable, other than the range (57) on $\omega_{g}$, the universe should be flat or positively curved $(k \geq 0)$ for a constant curvature 
bulk, i.e for $\mu=0$. In the case of the existence of a non-constant curvature bulk, for $\mu>0$ we require $k>0$ while for $\mu<0$, the zero, positive and negative values for $k$ are allowed by setting the appropriate numerical values for the parameters $k, a_{0}$ and $\mu$. Then, for a flat universe, as confirmed by the current observations, a bulk space with negative curvature parameter $\mu$ is needed. Moreover, regarding the values of parameters $k, a_{0}, b_{0}$ and $\mu$, for the case of $k a_{0}^{2}-\mu<b_{0}^{2}$, we have the total range $-\frac{5}{3}<\omega_{g}<-\frac{1}{3}$ which represents that the geometric equation of state parameter completely lies in the strong energy condition (SEC) violating range while for the case of $k a_{0}^{2}-\mu>b_{0}^{2}$ it may include a range of normal matter respecting the SEC.

\subsubsection{Radiation dominated state $\omega=\frac{1}{3}$.}

For this case, the acceptable range is

$$
-\frac{1}{3}-\frac{2}{3} \sqrt{1-\frac{k a_{0}^{2}-\frac{1}{3} \mu}{b_{0}^{2}}}<\omega_{g}<-\frac{1}{3}+\frac{2}{3} \sqrt{1-\frac{k a_{0}^{2}-\frac{1}{3} \mu}{b_{0}^{2}} .}
$$

It is also seen that we should have

$$
1-\frac{k a_{0}^{2}-\frac{1}{3} \mu}{b_{0}^{2}} \geq 0
$$

which gives a restriction on the scale factor of Einstein static universe $a_{0}$, the brane curvature warp $b_{0}$ and bulk curvature parameter $\mu$. Regarding the constraint (60), and in a constant curvature bulk $(\mu=0)$, for the case of flat and positive curvature universe $(k \geq 0)$, we have the total range $-1<\omega_{g}<\frac{1}{3}$ representing that the geometrical equation of state parameter includes both of the normal and SEC violating range. For a non-constant curvature bulk, deducing such a total range for $\omega_{g}$ requires the numerical values of $k, a_{0}, b_{0}$ and $\mu$. However, for a spatially flat universe, the bulk space can take both of the positive and negative curvature parameter $\mu$ regarding the value of curvature warp of the brane $b_{0}$ satisfying relation (601). 


\subsubsection{Matter dominated state, $\omega=0$}

The acceptable region for $\omega_{g}$ is

$$
-\frac{1}{2}-\frac{1}{2} \sqrt{1-\frac{8 k a_{0}^{2}}{9 b_{0}^{2}}}<\omega_{g}<-\frac{1}{2}+\frac{1}{2} \sqrt{1-\frac{8 k a_{0}^{2}}{9 b_{0}^{2}}}
$$

where there is also an additional condition on the scale factor of Einstein static universe $a_{0}$ and the curvature warp $b_{0}$ as

$$
1-\frac{8 k a_{0}^{2}}{9 b_{0}^{2}} \geq 0
$$

implying that the universe can be flat, positively or negatively curved depending on the values of the scale factor of Einstein static universe $a_{0}$ and the curvature warp $b_{0}$. For the flat and positively curved universe $(k \geq 0)$, regarding the constraint (62) and depending on the values of $k, a_{0}$ and $b_{0}$, the total range for the geometric equation of state parameter will be $-1<\omega_{g}<0$ representing that the geometrical equation of state parameter includes both of the normal and SEC violating range. For the case of $k<0$, deducing such a total range for $\omega_{g}$ requires the numerical values of the parameters $k, a_{0}$ and $b_{0}$ but a larger range including both of the SEC respecting and violating range is obtainable.

\subsection{The case of $A_{i}=B=0$.}

The acceptable region for $\omega_{g}$ is

$$
-\frac{1}{2}-\frac{1}{2} \sqrt{1-\frac{8 k a_{0}^{2}-16 \mu}{9 b_{0}^{2}}}<\omega_{g}<-\frac{1}{2}+\frac{1}{2} \sqrt{1-\frac{8 k a_{0}^{2}-16 \mu}{9 b_{0}^{2}}}
$$

where there is also an additional condition on the scale factor of Einstein static universe $a_{0}$, the curvature warp $b_{0}$ and bulk curvature parameter $\mu$ as

$$
1-\frac{8 k a_{0}^{2}-16 \mu}{9 b_{0}^{2}} \geq 0
$$


For $\mu=0$, this case corresponds to a universe supported just by dust matter on the brane with a geometrical induced matter. Using relations (51)-(55), this case has the acceptable region as the same as the above matter dominated state, $\omega=0$. Then, for this case, the stable ESU demands an equation of state parameter as the equations (61) and (62). For a non-constant curvature bulk with $\mu<0$ and $k>0$, we obtain the total range as $-1<\omega_{g}<0$ including both of the SEC respecting and violating range. For $\mu>0$ deducing a range for $\omega_{g}$ requires the numerical values of $k, a_{0}, b_{0}$ and $\mu$.

\subsection{The case of $A_{i}=0$.}

This case is known as the generalized Chaplygin gas model introduced in Kamenshchik et al (2001) and elaborated in (Bento et al 2002). For this case, we obtain

$$
\begin{aligned}
& \omega_{g}^{(1)}=-\frac{1}{2}+\frac{\alpha B}{2 \rho_{0}^{\alpha-1}}-\frac{1}{2} \sqrt{\left(1-\frac{\alpha B}{\rho_{0}^{\alpha-1}}\right)^{2}-4\left[\frac{2 k a_{0}^{2}-4 \mu}{9 b_{0}^{2}}-\frac{\alpha B}{\rho_{0}^{\alpha-1}}\left(1-\frac{6 k a_{0}^{2}+4 \mu}{9 b_{0}^{2}}\right)\right]} \\
& \omega_{g}^{(2)}=-\frac{1}{2}+\frac{\alpha B}{2 \rho_{0}^{\alpha-1}}+\frac{1}{2} \sqrt{\left(1-\frac{\alpha B}{\rho_{0}^{\alpha-1}}\right)^{2}-4\left[\frac{2 k a_{0}^{2}-4 \mu}{9 b_{0}^{2}}-\frac{\alpha B}{\rho_{0}^{\alpha-1}}\left(1-\frac{6 k a_{0}^{2}+4 \mu}{9 b_{0}^{2}}\right)\right]}
\end{aligned}
$$

where we also should have

$$
\alpha^{2}+2 \frac{\rho_{0}^{\alpha-1}}{B}\left[1-\frac{12 k a_{0}^{2}+8 \mu}{9 b_{0}^{2}}\right] \alpha+\frac{\rho_{0}^{2(\alpha-1)}}{B^{2}}\left(1-\frac{8 k a_{0}^{2}-16 \mu}{9 b_{0}^{2}}\right) \geq 0
$$

which confines the acceptable range for $\alpha$ as

$$
\alpha \leq \alpha^{(1)} \quad \& \quad \alpha \geq \alpha^{(2)}
$$

where

$$
\begin{aligned}
& \alpha^{(1)}=-\frac{1}{2} \mathcal{M}_{1}-\frac{1}{2} \sqrt{\mathcal{M}_{1}^{2}-4 \mathcal{M}_{2}} \\
& \alpha^{(2)}=-\frac{1}{2} \mathcal{M}_{1}+\frac{1}{2} \sqrt{\mathcal{M}_{1}^{2}-4 \mathcal{M}_{2}}
\end{aligned}
$$


and

$$
\begin{aligned}
& \mathcal{M}_{1}=2 \frac{\rho_{0}^{\alpha-1}}{B}\left[1-\frac{12 k a_{0}^{2}+8 \mu}{9 b_{0}^{2}}\right] \\
& \mathcal{M}_{2}=\frac{\rho_{0}^{2(\alpha-1)}}{B^{2}}\left(1-\frac{8 k a_{0}^{2}-16 \mu}{9 b_{0}^{2}}\right) .
\end{aligned}
$$

Generally, deducing a total acceptable range or a specific value for $\omega_{g}$ requires the values of parameters $k, a_{0}, b_{0}, \rho_{0}, \alpha, \mu$ and $B$. However, it is interesting that for the case of constant curvature bulk and flat universe, i.e $k=0=\mu$, we have

$$
\begin{aligned}
& \omega_{g}^{(1)}=-1, \\
& \omega_{g}^{(2)}=\frac{\alpha B}{\rho_{0}^{\alpha-1}},
\end{aligned}
$$

which using the relation (53) gives a restriction on $\alpha$ and $B$ values of the model as $\alpha B>-\rho_{0}^{\alpha-1}$. For this case we also find

$$
\alpha^{(1)}=\alpha^{(2)}=-\frac{\rho_{0}^{\alpha-1}}{B}
$$

Consequently, because of weak energy condition which imposes a positive energy density, the $\alpha$ parameter can take any positive or negative values with respect to the $B$ values. Interestingly, for the case $\alpha=-\frac{\rho_{0}^{\alpha-1}}{B}$, we have $\omega_{g}=\omega_{g}^{(1)}=\omega_{g}^{(2)}=-1$ which denotes that the geometric fluid behaves as the cosmological constant.

\subsection{The case of $A_{i}=0$ with $\alpha=1$.}

This case represents the standard Chaplygin gas model on the brane. For this case we have

$$
\begin{aligned}
& \omega_{g}^{(1)}=-\frac{1}{2}+\frac{B}{2}-\frac{1}{2} \sqrt{(1-B)^{2}-4\left[\frac{2 k a_{0}^{2}-4 \mu}{9 b_{0}^{2}}-B\left(1-\frac{6 k a_{0}^{2}+4 \mu}{9 b_{0}^{2}}\right)\right]}, \\
& \omega_{g}^{(2)}=-\frac{1}{2}+\frac{B}{2}+\frac{1}{2} \sqrt{(1-B)^{2}-4\left[\frac{2 k a_{0}^{2}-4 \mu}{9 b_{0}^{2}}-B\left(1-\frac{6 k a_{0}^{2}+4 \mu}{9 b_{0}^{2}}\right)\right]},
\end{aligned}
$$


which has interesting solutions for $B=1$ and $B=\frac{1}{3}$. For $B=1$, we have

$$
\begin{aligned}
& \omega_{g}^{(1)}=-\sqrt{1-\frac{8 k a_{0}^{2}}{9 b_{0}^{2}}}, \\
& \omega_{g}^{(2)}=\sqrt{1-\frac{8 k a_{0}^{2}}{9 b_{0}^{2}}},
\end{aligned}
$$

with the additional condition as $1-\frac{8 k a_{0}^{2}}{9 b_{0}^{2}}>0$. It is interesting that the effect of bulk curvature does not appear in this case. For the spatially flat and positively curved universe, we obtain the total range for $\omega_{g}$ as $-1<\omega_{g}<1$ including both of the SEC respecting and violating range. Similarly, for the case of $B=\frac{1}{3}$, we obtain

$$
\begin{aligned}
& \omega_{g}^{(1)}=-\frac{1}{3}-\frac{2}{3} \sqrt{1-\frac{3 k a_{0}^{2}-2 \mu}{3 b_{0}^{2}}}, \\
& \omega_{g}^{(2)}=-\frac{1}{3}+\frac{2}{3} \sqrt{1-\frac{3 k a_{0}^{2}-2 \mu}{3 b_{0}^{2}}},
\end{aligned}
$$

which requires $1-\frac{3 k a_{0}^{2}-2 \mu}{3 b_{0}^{2}} \geq 0$. For a constant curvature bulk with spatially flat and positively curved brane universe, i.e for $\mu=0$ with $k \geq 0$, we obtain the total range as $-1<\omega_{g}<\frac{1}{3}$ including both of the SEC respecting and violating range. For a non-constant curvature bulk with $\mu<0$ and $k>0$, we also obtain the total range as $-1<\omega_{g}<\frac{1}{3}$ including both of the SEC respecting and violating range. For $\mu>0$ deducing a range for $\omega_{g}$ requires the numerical values of $k, a_{0}, b_{0}$ and $\mu$.

\section{Final Remarks}

A cosmological model in which an extended Chaplygin gas universe, with the equation

of state $p=\sum_{i=1}^{n} A_{i} \rho^{i}-\frac{B}{\rho^{\alpha}}$, is merged with the braneworld scenario has been investigated in this work. A general stability condition for the Einstein static state on the brane embedded in a general non-constant curvature bulk space has been obtained. Moreover, subsets of 
generalized and standard Chaplygin gas model, brane with dust matter and with barotropic equation of state solutions have been separately discussed. For each case, we have obtained the stability conditions and their impacts on the geometric equation of state parameter $\omega_{g}$ as well as the spatial curvature $k$ of the universe in terms of the scale factor of Einstein static universe $a_{0}$, the brane curvature warp $b_{0}$ factor and bulk space curvature parameter $\mu$. In the following, some of our results are represented.

For the case of $B=0$ with $A_{i \geq 2}=0$ and defining $A_{1}=\omega$, we are reduced to the barotropic equation of state. Then, we analyze the vacuum dominated state $(\omega=-1)$, the radiation dominated state $\left(\omega=\frac{1}{3}\right)$ and matter dominated state $(\omega=0)$ in both of the constant and non-constant curvature bulk space. For the case of the vacuum dominated state with constant curvature bulk $(\mu=0)$, the universe should be flat or positively curved $(k \geq 0)$. In the case of the non-constant curvature bulk, for $\mu>0$ we require $k>0$ while for $\mu<0$, the zero, positive and negative values for $k$ are allowed. Moreover, respecting to the values of parameters $k, a_{0}, b_{0}$ and $\mu$, for the case of $k a_{0}^{2}-\mu<b_{0}^{2}$, we have the total range $-\frac{5}{3}<\omega_{g}<-\frac{1}{3}$ which represents that the geometric equation of state parameter completely lies in the strong energy condition (SEC) violating range while for the case of $k a_{0}^{2}-\mu>b_{0}^{2}$ it may include a range of normal matter respecting the SEC. For the radiation dominated state with $\mu=0$ and $k \geq 0$, we have the total range $-1<\omega_{g}<\frac{1}{3}$ representing that the geometrical equation of state parameter includes both of the normal and SEC violating range. In the case of matter dominated state with $k \geq 0$, the total range is obtained as $-1<\omega_{g}<0$ representing that the geometrical equation of state parameter includes both of the normal and SEC violating range.

For the case of $A_{i}=B=0$ with $\mu=0$, the acceptable region for $\omega_{g}$ is the same as the matter dominated state $\omega=0$ while for a non-constant curvature bulk with $\mu<0$ and $k>0$, the total range is obtained as $-1<\omega_{g}<0$ including both of the SEC respecting and 
violating range.

For the case of $A_{i}=0$ which is known as the generalized Chaplygin gas model, a total range for $\omega_{g}$ is obtained. Specifically, it is shown that for the case of constant curvature bulk and flat universe $(k=0=\mu)$, the geometric fluid behaves as the cosmological constant, i.e $\omega_{g}=-1$

For the case of $A_{i}=0$ with $\alpha=1$, representing the standard Chaplygin gas model on the brane, a total range for $\omega_{g}$ is also obtained. Interestingly, it is shown that by setting $B=1$, the effect of bulk curvature does not appear in the acceptable range of $\omega_{g}$. For $k \geq 0$, the total range as $-1<\omega_{g}<1$ including both of the SEC respecting and violating range is obtained. Also, by setting $B=\frac{1}{3}$, for $\mu=0$ with $k \geq 0$, the total range as $-1<\omega_{g}<\frac{1}{3}$ including both of the SEC respecting and violating range is obtained. Finally, for a non-constant curvature bulk with $\mu<0$ and $k>0$, we also obtain the total range as $-1<\omega_{g}<\frac{1}{3}$ including both of the SEC respecting and violating range.

\section{Acknowledgment}

This work has been supported financially by Research Institute for Astronomy and Astrophysics of Maragha (RIAAM) under research project NO.1/4165-92. 


\section{REFERENCES}

A. Ali, S. Dutta, E. N. Saridakis and A. A. Sen, Gen. Rel. Grav. 44, 657 (2012) arXiv:1004.2474 [astro-ph.CO]].

L. Amendola, R. Gannouji, D. Polarski and S. Tsujikawa, Phys. Rev. D 75, 083504 (2007) gr-qc/0612180.

C. Armendariz-Picon, V. F. Mukhanov and P. J. Steinhardt, Phys. Rev. D 63, 103510 (2001) astro-ph/0006373.

K. Atazadeh, JCAP 06, 020 (2014).

K. Atazadeh and F. Darabi, Eur. Phys. J. C 72, 2016 (2012) arXiv:1112.2824 [physics.gen$\mathrm{ph}]]$.

K. Atazadeh and F.darabi, Phys. Lett. B 744, 363 (2015).

K. Atazadeh, Y. Heydarzade and F. Darabi, Phys. Lett. B 732, 223 (2014).

K. Bamba, S. Capozziello, S. Nojiri, S. D. Odintsov, Astrophys. Space Sci. 342, 155 (2012).

J. D. Barrow and A. C. Ottewill, J. Phys. A 16, 2757 (1983).

D. Bazeia, A. R. Gomes, L. Losano and R. Menezes, Phys. Lett. B 671, 402 (2009) arXiv:0808.1815 [hep-th]].

G. R. Bengochea and R. Ferraro, Phys. Rev. D 79, 124019 (2009) arXiv:0812.1205 [astro-ph]].

M. C. Bento, O. Bertolami and A. A. Sen, Phys. Rev. D 66, 043507 (2002) gr-qc/0202064.

N. Bilic, G. B. Tupper and R. D. Viollier, Phys. Lett. B 535, 17 (2002) astro-ph/0111325.

C. G. Bohmer, Class. Quant. Grav. 21, 1119 (2004). 
C. G. Bohmer, L. Hollenstein and F. S. N. Lobo, Phys. Rev. D 76, 084005 (2007).

P. Brax and C. van de Bruck, Class. Quant. Grav. 20, R201 (2003) [hep-th/0303095].

Y. F. Cai, E. N. Saridakis, M. R. Setare and J. Q. Xia, Phys. Rept. 493, 1 (2010) arXiv:0909.2776 [hep-th]].

G. Calcagni, JHEP 0909, 112 (2009) arXiv:0904.0829 [hep-th]].

R. R. Caldwell, Phys. Lett. B 545, 23 (2002) astro-ph/9908168.

R. R. Caldwell, M. Kamionkowski and N. N. Weinberg, Phys. Rev. Lett. 91, 071301 (2003) astro-ph/0302506.

R. Canonico and L. Parisi, Phys. Rev. D 82, 064005 (2010).

S. Capozziello, V. F. Cardone and A. Troisi, Phys. Rev. D 71, 043503 (2005) astro-ph/0501426.

S. Capozziello, F. Darabi and D. Vernieri, Mod. Phys. Lett. A 26, 65 (2011) arXiv:1006.0454 $[\mathrm{gr}-\mathrm{qc}]]$.

S. Capozziello and M. De Laurentis, Phys. Rept. 509, 167 (2011) [arXiv:1108.6266 [gr-qc]].

C. Clarkson and S. S. Seahra, Class. Quant. Grav. 22, 3653 (2005).

E. J. Copeland, M. Sami and S. Tsujikawa, Int. J. Mod. Phys. D 15, 1753 (2006) hep-th/0603057.

F. Darabi, Y. Heydarzade and F. Hajkarim, arXiv:1406.7636 [hep-th]].

F. Darabi, W. N. Sajko and P. S. Wesson, Class. Quant. Grav. 17, 4357 (2000) gr-qc/0005036.

A. De Felice and S. Tsujikawa, Living Rev. Rel. 13, 3 (2010) arXiv:1002.4928 [gr-qc]]. 
A. De Felice and S. Tsujikawa, Phys. Lett. B 675, 1 (2009).

C. Deffayet, G. Esposito-Farese and A. Vikman, Phys. Rev. D 79, 084003 (2009) arXiv:0901.1314 [hep-th]].

C. Deffayet, X. Gao, D. A. Steer and G. Zahariade, Phys. Rev. D 84, 064039 (2011) arXiv:1103.3260 [hep-th]]. arXiv:0810.5712 [hep-th]].

S. Dutta, E. N. Saridakis and R. J. Scherrer, Phys. Rev. D 79, 103005 (2009) [arXiv:0903.3412 [astro-ph.CO]].

N. Deruelle and L. Farina-Busto, Phys. Rev. D 41, 3696 (1990).

L. P. Eisenhart, Riemannian Geometry, Princeton University Press, Princeton NJ (1966).

G. F. R. Ellis and R. Maartens, Class. Quant. Grav. 21, 223 (2004).

G. F. R. Ellis, J. Murugan and C. G. Tsagas, Class. Quant. Grav. 21, 233 (2004).

E. E. Flanagan, Phys. Rev. D 74, 023002 (2006) astro-ph/0605504.

L. Gergely and R. Maartens, Class. Quant. Grav. 19, 213 (2002).

N. Goheer, R. Goswami, and P. K. S. Dunsby, Class. Quant. Grav. 26, 105003 (2009).

V. Gorini, A. Kamenshchik and U. Moschella, Phys. Rev. D 67, 063509 (2003) astro-ph/0209395].

R. Goswami, N. Goheer and P. K. S. Dunsby, Phys. Rev. D 78, 044011 (2008).

Z. K. Guo, N. Ohta and Y. Z. Zhang, Mod. Phys. Lett. A 22, 883 (2007) astro-ph/0603109.

Z. K. Guo, Y. S. Piao, X. M. Zhang and Y. Z. Zhang, Phys. Lett. B 608, 177 (2005) astro-ph/0410654. 
M. Heydari-Fard and H. R. Sepangi, Phys. Rev. D 76, 104009 (2007) arXiv:0710.2666 $[\mathrm{gr}-\mathrm{qc}]]$.

M. Heydari-Fard and H. R. Sepangi, Phys. Rev. D 78, 064007 (2008) arXiv:0809.0546 $[\mathrm{gr}-\mathrm{qc}]]$.

Y. Heydarzade and F. Darabi, JCAP 04, 028 (2015) arXiv:1501.02624 [gr-qc]].

Y. Heydarzade, M. Khodadi and F. Darabi, arXiv:1502.04445 [gr-qc]].

K. Hinterbichler, Rev. Mod. Phys. 84, 671 (2012) [arXiv:1105.3735 [hep-th]].

G. Hooft, gr-qc/9310026.

P. Horava, JHEP 0903, 020 (2009) arXiv:0812.4287 [hep-th]].

G. W. Horndeski, Int. J. Theor. Phys. 10, 363-384 (1974).

K. Ichiki, M. Yahiro, T. Kajino, M. Oritoand and G. J. Mathews, Phys. Rev. D 66, 043521 (2002).

S. Jalalzadeh and H. R. Sepangi, Class. Quant. Grav 22, 2035 (2005).

E. O. Kahya and B. Pourhassan, Astrophys. Space Sci. 353, 677 (2014).

A. Kamenshchik, U. Moschella, and V. Pasquier, Phys. Lett. B 511, 265 (2001).

M. Khodadi, Y. Heydarzade, K. Nozari and F. Darabi, arXiv:1505.00342v2 [gr-qc]].

K. Koyama, Gen. Rel. Grav. 40, 421 (2008).

Langlois, R. Maartens, M. Sasaki, and D. Wands, Phys. Rev. D 63, 084009 (2001).

G. Leon and E. N. Saridakis, JCAP 1303, 025 (2013) [arXiv:1211.3088 [astro-ph.CO]].

G. Leon, J. Saavedra and E. N. Saridakis, Class. Quant. Grav. 30, 135001 (2013). 
J. T. Li, C. C. Lee,C. Q. Geng, Eur. Phys. J. C 73, 2315 (2013).

A. R. Liddle and R. J. Scherrer, Phys. Rev. D 59, 023509 (1999).

E. V. Linder, Phys. Rev. D 81, 127301 (2010); Phys. Rev. D 82, 109902 (2010).

L. Lombriser, W. Hu, W. Fang and U. Seljak, Phys. Rev. D 80, 063536 (2009).

D. Lovelock, J. Math. Phys. 12, 498 (1971).

J. Lu, L. Xu, H. Tan and S. Gao, Phys. Rev. D 89, 063526 (2014).

H. Maeda, V. Sahni and Y. Shtanov, Phys. Rev. D 76, 104028 (2007); Phys. Rev. D 80, $089902(2009)$.

M. D. Maia, E. M. Monte, J. M. F. Maia and J. S. Alcaniz, Class. Quant. Grav. 22, 1623 $(2005)$.

M. D. Maia and W. L. Roque, Phys. Lett. A 139, 121 (1989).

P. D. Mannheim and D. Kazanas, Astrophys. J. 342, 635 (1989).

S. Mukherjee, B. C. Paul, N. K. Dadhich, S. D. Maharaj and A. Beesham, Class. Quant. Grav. 23, 6927 (2006).

S. Mukohyama, Phys. Lett. B 473, 241 (2000).

D. J. Mulryne, R. Tavakol, J. E. Lidsey and G. F. R. Ellis, Phys. Rev. D 71, 123512 (2005).

J. Nash, Ann. Math, 63, 20 (1956).

A. Nicolis, R. Rattazzi and E. Trincherini, Phys. Rev. D 79, 064036 (2009).

S. Nojiri and S. D. Odintsov, Phys. Lett. B 562, 147 (2003).

S. Nojiri and S. D. Odintsov, Gen. Rel. Grav. 38, 1285 (2006). 
S. Nojiri and S. D. Odintsov, Phys. Lett. B 631, 1 (2005).

S. Nojiri and S. D. Odintsov, eConf C 0602061, 06 (2006) Int. J. Geom. Meth. Mod. Phys. 4, 115 (2007).

V. K. Onemli and R. P. Woodard, Phys. Rev. D 70, 107301 (2004).

A. Paliathanasis, S. Basilakos, E. N. Saridakis, S. Capozziello, K. Atazadeh, F. Darabi and M. Tsamparlis, Phys. Rev. D 89, no. 10, 104042 (2014).

L. Parisi, N. Radicella and G. Vilasi, Phys. Rev. D 86, 024035 (2012).

L. Parisi, N. Radicella and G. Vilasi, Springer Proc. Math. Stat 60, 355 (2014).

L. Parisi, M. Bruni, R. Maartens and K. Vandersloot, Class. Quant. Grav. 24, 6243 (2007).

P. J. E. Peebles and B. Ratra, Rev. Mod. Phys. 75, 559 (2003).

S. Perlmutter et al., Astrophys. J. 517, 565 (1999).

B. Pourhassan, Int. J. Mod. Phys. D 22, 1350061 (2013).

B. Pourhassan and E. O. Kahya, Adv. High Energy Phys. 2014, 231452 (2014) arXiv:1405.0667 [gr-qc]].

L. Randall and R. Sundrum, Phys. Rev. Lett. 83, 3370 (1999) hep-ph/9905221.

A. R. Rastkar, M. R. Setare and F. Darabi, Astrophys. Space Sci. 337, 487 (2012) arXiv:1104.1904 [gr-qc]].

B. Ratra and P. J. E. Peebles, Phys. Rev. D 37, 3406 (1988).

C. de Rham, G. Gabadadze and A. J. Tolley, Phys. Rev. Lett. 106, 231101 (2011) arXiv:1011.1232 [hep-th]]. 
C. de Rham, Living Rev. Rel. 17, 7 (2014) arXiv:1401.4173 [hep-th]].

A. G. Riess et al., Astron. J. 116, 1009 (1998) astro-ph/9805201.

V. A. Rubakov and M. E. Shaposhnikov, Phys. Lett. B 125, 136 (1983).

E. N. Saridakis, Eur. Phys. J. C 67, 229 (2010) [arXiv:0905.3532 [hep-th]].

E. N. Saridakis, Phys. Lett. B 676, 7 (2009) [arXiv:0811.1333 [hep-th]].

S. S. Seahra and C. G. Bohmer, Phys. Rev. D 79, 064009 (2009).

M. R. Setare and E. N. Saridakis, JCAP 0903, 002 (2009) [arXiv:0811.4253 [hep-th]].

F. Schmidt, Phys. Rev. D 80, 043001 (2009) arXiv:0905.0858 [astro-ph.CO]].

L. Susskind, J. Math. Phys. 36, 6377 (1995).

C. Wetterich, Nucl. Phys. B 302, 668 (1988).

J. T. Wheeler, Nucl. Phys. B 268, 737 (1986).

P. Wu, H. Yu, Phys. Lett. B 703, 223 (2011).

P. Wu and H. Yu, Phys. Rev. D 81, 103522 (2010).

K. Zhang, P. Wu and H. Yu, Phys. Lett. B 690, 229 (2010).

K. Zhang, P. Wu and H. Yu, Phys. Rev. D 85, 043521 (2012).

K. Zhang, P. Wu, H. Yu, Phys. Rev. D 87, 063513 (2013).

W. Zhao, Phys. Rev. D 73, 123509 (2006) astro-ph/0604460. 